\title{
A novel proteotoxic stress associated mechanism for macular corneal dystrophy
}

\author{
Kai Kaarniranta ${ }^{1,2 \star}$, Eszter Szalai ${ }^{3 *}$, Adrian Smedowski ${ }^{4,5}$, Zoltán Hegyi ${ }^{6}$, Niko Kivinen ${ }^{1}$, Johanna Viiri ${ }^{1}$, Bogumil \\ Wowra $^{4}$, Dariusz Dobrowolski ${ }^{4}$, László Módis $\mathrm{Jr}^{3}{ }^{3}$, András Berta ${ }^{3}$, Edward Wylegala ${ }^{4}$ and Szabolcs Felszeghy ${ }^{6}$ \\ ${ }^{1}$ Department of Ophthalmology, Institute of Clinical Medicine, University of Eastern Finland, Kuopio, Finland, ${ }^{2}$ Department of \\ Ophthalmology, Kuopio University Hospital, Kuopio, Finland, ${ }^{3}$ Department of Ophthalmology, University of Debrecen, Medical \\ Faculty, Debrecen, Hungary, ${ }^{4} \mathrm{Clinical}$ Department of Ophthalmology, Faculty of Medicine and Division of Dentistry in Zabrze, Medical \\ University of Silesia, Katowice, Poland, ${ }^{5}$ Department of Physiology, Medical University of Silesia, Katowice, Poland and ${ }^{6}$ Department \\ of Anatomy, Histology and Embryology, University of Debrecen, Medical Faculty, Debrecen, Hungary \\ *equal contribution
}

Summary. Macular corneal dystrophy is a rare autosomal recessive eye disease affecting primarily the corneal stroma. Abnormal accumulation of proteoglycan aggregates has been observed intra- and extracellularly in the stromal layer. In addition to the stromal keratocytes and corneal lamellae, deposits are also present in the basal epithelial cells, endothelial cells and Descemet's membrane. Misfolding of proteins has a tendency to gather into aggregating deposits. We studied interaction of molecular chaperones and proteasomal clearance in macular dystrophy human samples and in human corneal HCE-2 epithelial cells. Seven cases of macular corneal dystrophy and four normal corneal buttons collected during corneal transplantation were examined for their expression patterns of heat shock protein 70 , ubiquitin protein conjugates and SQSTM1/p62. In response to proteasome inhibition the same proteins were analyzed by western blotting. Slitlamp examination, in vivo confocal cornea microscopy and transmission electron microscopy were used for morphological analyses. Heat shock protein 70 , ubiquitin protein conjugates and SQSTM1/p62 were upregulated in both the basal corneal epithelial cells and the stromal keratocytes in macular corneal dystrophy samples that coincided with an increased expression of the same molecules under proteasome inhibition in the HCE-2 cells in vitro. We propose a novel regulatory mechanism

Offprint requests to: Professor Kai Kaarniranta, Department of Ophthalmology, Kuopio University Hospital, P.O. Box 1777, FIN-70211, Kuopio, Finland. e-mail: kai.kaarniranta@uef.fi

DOI: $10.14670 / \mathrm{HH}-11-588$ that connects the molecular chaperone and proteasomal clearance system in the pathogenesis of macular corneal dystrophy.

Key words: Heat shock proteins, Macular corneal dystrophy, Misfolding, Protein aggregation, Ubiquitin/ proteasome pathway

\section{Introduction}

Macular corneal dystrophy (Groenouw corneal dystrophy type II, MIM \#217800) is an uncommon, autosomal recessive disorder affecting the corneal stroma (Weiss et al., 2008). Most cases are linked to mutations in the carbohydrate sulfotransferase gene (CHST6) on chromosome 16q22 (Akama et al., 2000). Macular corneal dystrophy (MCD) has been classified as immunophenotype I, IA and II based on sulfated keratan sulfate level of the serum and cornea (Klintworth et al., 1986, 1997). It is characterized by abnormal accumulation of proteoglycan aggregates intra- and extracellularly in the stromal layer resulting in various irregular gray-white opacities extending to the peripheral and posterior stroma (Klintworth and Smith, 1977; Jonasson et al., 1989; Klintworth, 2009). In addition to the keratocytes and corneal lamellae, deposits are also present in the basal epithelial cells, endothelial cells and Descemet's membrane, and recurrent epithelial erosions are seen. As the disease progresses, the aggregates become more confluent with diffuse corneal haze. Macular corneal dystrophy is accompanied by decreased 
corneal thickness. This condition may lead to severe bilateral visual impairment until the fourth-fifth decade of life (Klintworth, 2009).

Misfolded proteins have a tendency to gather into detrimental aggregates (Tyedmers et al., 2010). In addition to stress-damaged proteins, the nonfunctional proteins may also be a result of gene mutations and disturbances in protein synthesis leading to misfolding and aggregation (Goldberg, 2003). Prior to the ultimate aggregation cellular defense mechanism is triggered, called the molecular chaperone response (Heat shock protein response; Hsp response), which attempts to restore proteins to their original folding state, retain their function and reduce the aggregation process (Kästle and Grune, 2012). If this response is unsuccessful, misfolded soluble proteins are tagged with a small polypeptide ubiquitin ( $\mathrm{Ub}$ ) that directs the complex to the ubiquitin/proteasomal protein degradation pathway (UPP) (Jung et al., 2009). The eukaryotic proteasome is a multicatalytic proteolytic complex present in cytosol, nucleus and microsomes (Kopito, 2000). The proteasome recognizes and selectively degrades ubiquitinated proteins. The UPP is one of the most important cellular systems to remove misfolded proteins. Accumulation of ubiquinated proteins is a hallmark of impaired clearance via proteasomes that may lead to protein aggregation intra- and extracellularly (Carrard et al., 2002). The ability of SQSTM1/p62 to bind noncovalently to ubiquitin was first described by Vadlamudi et al. (1996), but only later was it realized that SQSTM1/p62 is one of the main molecules controlling the shuttling of ubiquitinated substrates towards proteasomal degradation (Janse et al., 2004; Seibenhener et al., 2004; Babu et al., 2005).

Since MCD is clearly associated to the aggregation process, our aim was to study the role of proteasomal clearance in the MCD pathology. Here we show for the first time that $\mathrm{Hsp} 70$, Ub protein conjugates and SQSTM1/p62 and protein aggregates are highly upregulated in both corneal basal epithelium and stroma cells of MCD samples and in response to proteasomal inhibition in corneal HCE-2 epithelial cells. Thus, we strongly believe that impaired UPP clearance is an important regulatory mechanism factor in the pathogenesis of MCD and may open novel ways to understand crosstalk of corneal epithelium and stroma.

\section{Materials and methods}

\section{Tissue samples}

Tissue samples were obtained from 7 patients who underwent penetrating keratoplasty. Donor cornea not used for corneal transplantation were obtained from the Cornea Bank Debrecen (Department of Ophthalmology, University of Debrecen /UD/, Medical and Health Science Centre /MHSC/) served as normal control corneas. MCD samples were obtained from the Department of Ophthalmology, UD MHSC and the Eye
Tissue Bank in Katowice (Ophthalmology Clinic, the Central Railway Hospital, Medical University of Silesia in Katowice). The analysis was performed on 50 sections derived from 11 corneas (7 MCD corneas of 7 patients with a mean age of $45 \pm 17$ years; 5 males, 2 females and 4 control corneas of 2 patients with a mean age of $51 \pm 8$ years; 1 male, 1 female). The MCD diagnosis was based on clinical signs and the family history questionnaire.

\section{In vivo corneal confocal microscopy}

Patients scheduled for corneal transplantation (from which tissue samples for further analysis were obtained) underwent in vivo corneal confocal microscopy (Rostock Cornea Module, Heidelberg Engineering Retina Tomograph III, Dossenheim, Germany). After topical anesthesia (Proxymetacaine hydrochloride, Alcon) scans of full thickness dystrophic corneas (from tear film to endothelium) were made. For this examination we used 2 different scanning frames - 300x300 $\mu \mathrm{m}$ and 400x400 $\mu \mathrm{m}$. Scans were made with $1 \mu \mathrm{m}$ step with continuous recording. For comparison, healthy corneas from 2 persons were examined.

\section{Laboratory procedures}

For histology and immunohistochemistry, corneal buttons were fixed in Saint-Marie solution (Saint-Marie, 1962; Felszeghy et al., 2004), dehydrated, and embedded in paraffin. Sections from $5 \mu \mathrm{m}$ to $7 \mu \mathrm{m}$ thickness were cut. All aspects of experimental procedures and protocols were approved by the Board of Scientific Committee of University of Debrecen and Katowice. Participants provided their written informed consent for the human material in this study.

\section{Histochemistry}

Alcian blue staining was carried out according to standard protocols and observed under a light microscope Nikon Eclipse 800 microscope equipped with 60x PanFlour Nikon objective (Nikon Corporation Instruments Company, Japan).

\section{Immunohistochemistry}

For multiple labeling combinations of 3 primary antibodies were applied as a cocktail diluted in blocking solution, as described below. In humidity chambers a mixture of antibodies were placed into sections, which contained anti-hsp70 (diluted 1:100 /ENZO, Farmingdale, NY, USA/), anti Ubiquitin (diluted 1:200 /DAKO, Golstrup, Denmark/) and SQSTM1/p62 (diluted 1:100/Progen, Heidelberg, Germany/). After 3 days incubation at $4^{\circ} \mathrm{C}$ the slides were rinsed in PBS and the mixture of secondary antibodies (Alexa Fluor 488 anti-mouse, Alexa Fluor 647 anti-guinea pig /Invitrogen, Camarillo, CA, USA/ and Alexa 546 anti rabbit 


\section{Proteotoxic stress in macular dystrophy}

/Molecular Probes, Garnd Island, NY, USA) was added for 2 hours at room temperature in 1/200 dilution. The slides were washed in PBS and the nuclei were stained using DAPI (4,6- diamidino-2-fenilindole, dihydrocloride; 1:1000/Molecular Probes, Camarillo, CA, USA/) in PBS containing $0.1 \%$ saponin for $30 \mathrm{~min}$ and covered with Vectashield mounting Media (Vector Laboratories, Burlingame, CA, USA). As negative controls for each double label, the same procedure as above was followed except that one of the primary antibodies was omitted.

\section{Confocal microscopy}

Image acquisition was performed on an Olympus FV1000 confocal laser scanning system built on an IX81 inverted microscope equipped with an ultraviolet laser diode (405 nm), a multiline Ar laser (458, 488 and 514 $\mathrm{nm})$, an $\mathrm{HeNe}(\mathrm{G})$ laser (543 nm) and an $\mathrm{HeNe}(\mathrm{R})$ laser (633 nm) (Olympus, Tokyo, Japan). Single $1 \mu \mathrm{m}$ thick optical sections were acquired using a 60x oil immersion objective (NA:1.42, Plan Apochromat).

DAPI was excited by a $405 \mathrm{~nm}$ laser diode and fluorescence was detected between 425-475 $\mathrm{nm}$. Alexa 488 was excited at $488 \mathrm{~nm}$ and fluorescence was detected in the $500-550 \mathrm{~nm}$ spectral region. Alexa 546 was excited at $543 \mathrm{~nm}$ and fluorescence was collected in the 555-655 nm spectral range. Alexa 647 was excited at $633 \mathrm{~nm}$, and the emission was detected through $640 \mathrm{~nm}$ long-pass filter.

The confocal settings (laser power, gain, offset, confocal aperture, pixel size) were identical for all scans and kept constant during imaging. Digital images were captured with Fluoview ASW 3.1 software and processed with Adobe Photoshop CS6 software. In all imaging processes, gamma adjustment was done in the whole image to maintain appropriate contrast.

\section{Cell culture and treatments}

Cells used in this study were cultured as recently documented (Kinnunen et al., 2013). Fresh medium was supplied to the cells every other day and the cells were subcultured twice a week using $0.25 \%$ trypsin-EDTA (cat. no. 25200056; Life Technologies, Invitrogen, Camarillo, CA, USA) to detach the cells from plates. In proteasome experiments, the cells were exposed to $1 \mu \mathrm{M}$ MG-132 proteasome inhibitor (Calbiochem, 474790) for 24 hours (h).

\section{Western blotting}

For western blotting, whole cell extracts $(15-20 \mu \mathrm{g}$ of protein) were run in $10 \%$ sodium dodecyl sulfate PAGE (PAGE; SDS-PAGE) gels and then wet-blotted to nitrocellulose membranes (Amersham, Pittsburgh, PA, USA). The whole cell extracts were prepared in MPERH (Mammalian Protein Extraction Reagent, Thermo Scientific,78501) according to the manufacturer's protocol. The membranes were blocked for $1 \mathrm{~h}$ in $3 \%$ fat-free dry milk in $0.3 \%$ Tween-20/phosphate buffered saline at room temperature (RT). Thereafter, the membranes were incubated for $1 \mathrm{~h}$ at RT with rabbit polyclonal ubiquitin antibody (cat. no. Z0458; DakoCytomation, Glostrup, Denmark), mouse monoclonal SQSTM1 (p62) antibody (cat. no. sc-28359; Santa Cruz Biotechnology, Santa Cruz, CA, USA), or mouse monoclonal Hsp70 antibody (StressGen, Ann Arbor, MI, USA). Primary antibodies were diluted $(1: 500,1: 2000$, or $1: 5000$, respectively) in $0.5 \%$ BSA (BSA) in $0.3 \%$ Tween-20/PBS. After washing $3 \mathrm{X}$ for 10 min with $0.3 \%$ Tween-20/PBS, the membranes were incubated for $1 \mathrm{~h}$ at RT with horseradish peroxidaseconjugated antirabbit IgG or antimouse IgG antibodies (GE Healthcare, Little Chalfont, Buckinghamshire, UK). The secondary antibodies were diluted for ubiquitin 1:15000, for p62 1:25000, and for Hsp70 1:20000 in 3\% fat-free dry milk in $0.3 \%$ Tween-20/PBS. Before detection, all of the membranes were washed as before. Protein-antibody-complexes were detected with an enhanced chemiluminescent assay for horseradish peroxidise (Millipore, Billerica, MA, USA). Experiments were performed in duplicate for each different cell preparation and $\alpha$-tubulin was used to normalize the data. Statistical analysis of western blot data was performed on the densitometric values obtained with the NIH image software 1.61 (downloadable at http://rsb.info.nih.gov/nih-image) and Quantity One ${ }^{\circledR}$ software.

\section{Transmission electron microscopy}

For transmission electron microscopy, the cells were treated with different concentrations of MG-132 for 24 h. Cell culture samples were prefixed with $2.5 \%$ glutaraldehyde in $0.1 \mathrm{M}$ phosphate buffer $\mathrm{pH} 7.4$ for $2 \mathrm{~h}$ at RT. After 15 min washing in $0.1 \mathrm{M}$ phosphate buffer, the samples were post-fixed in $1 \%$ osmium tetraoxide and $0.1 \mathrm{M}$ phosphate buffer for 1 hour, and again washed with phosphate buffer for $15 \mathrm{~min}$ prior to standard ethanol dehydration. Subsequently, the samples were infiltrated and embedded in LX-112 resin. Polymerization was carried out at $37^{\circ} \mathrm{C}$ for $24 \mathrm{~h}$ and at $60^{\circ} \mathrm{C}$ for $48 \mathrm{~h}$. The sections were examined with a JEM2100F transmission electron microscope (Jeol) at 200 $\mathrm{kV})$. Aggregates were manually counted. Eight cells were randomly selected from each group.

\section{Results}

Figure 1 shows the classical histological and clinical appearance of macular corneal dystrophy. Histological demonstration of Alcian blue staining indicates deposits within individual keratocytes and between the collagen fibers of the substantia propria in MCD buttons. In slitlamp examination stromal opacities with indistinct borders and intervening haze can be observed. In vivo corneal confocal microscopy analysis reveals 
abnormality in basal epithelial cell size and shape (Fig. $2)$. Cells become bigger and polygonal with bright contours and they show intracellular aggregates. In the anterior stroma hyperreflective keratocytes with vacuoles were observed (Fig. 3). We found that Hsp70, $\mathrm{Ub}$ and SQSTM1/p62, markers for impaired proteasomal clearance, are highly expressed in the basal epithelium of MCD samples (Fig. 4). A very weak Hsp70 immunostaining was mainly associated with the basal cells of corneal epithelium in contrast to macular dystrophy, where intense Hsp70 signals were recorded throughout the cytoplasm of basal corneal epithelial cells (Fig. 4A,B). In healthy controls Ub was only detected around some cell nuclei of basal cells (Fig. 4C). In MCD corneal buttons strong ubiquitin signals were obtained from corneal epithelial cells (Fig. 4D). In healthy corneas no SQSTM1/p62 immunoreactivity was observed (Fig. 4E), whereas faintly elevated
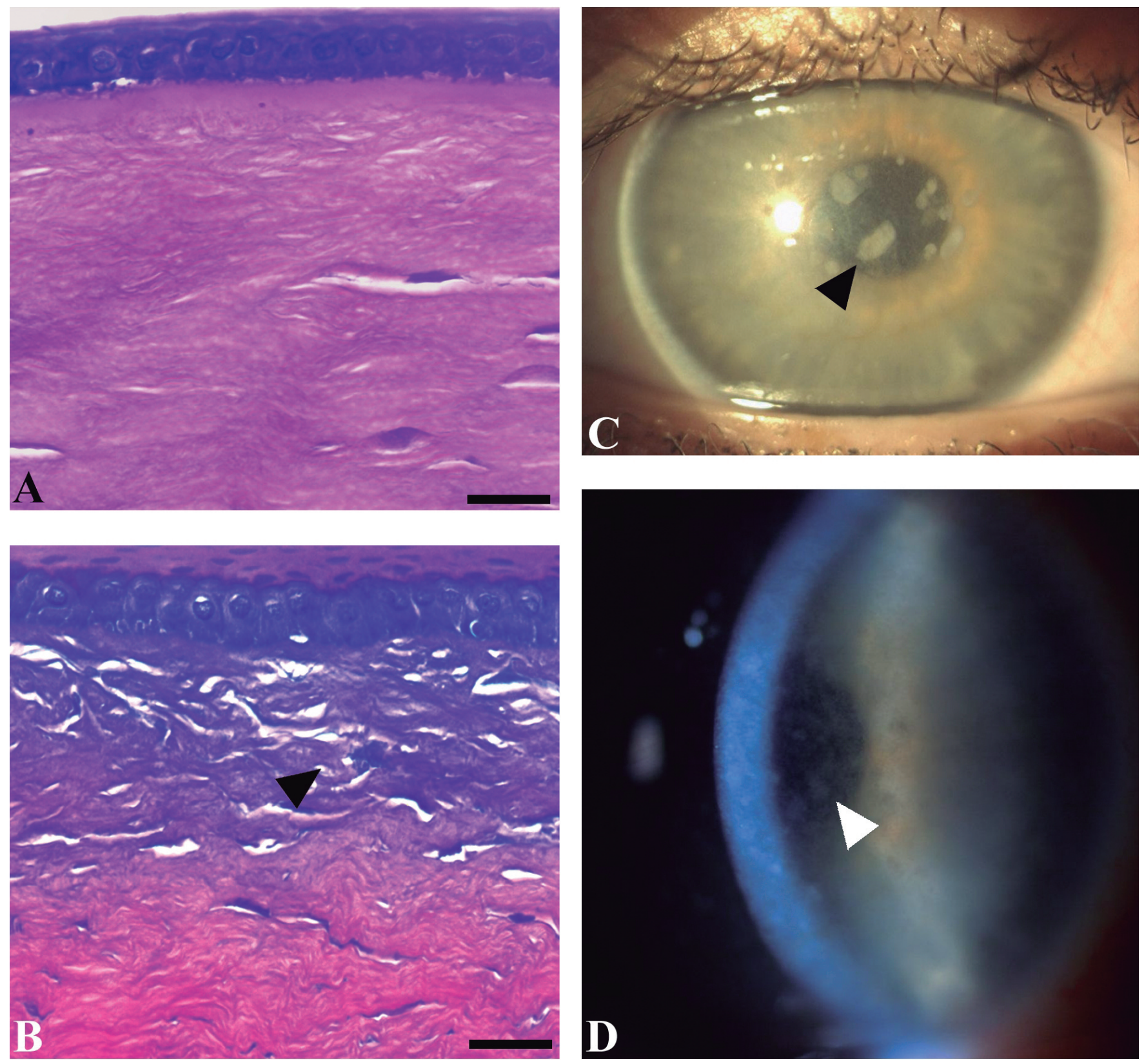

Fig. 1. Histological and clinical examination of cornea. Alcian blue staining for (A) normal human cornea and (B) MCD. Stromal deposits can be seen in slit-lamp examination (C, D). Arrowheads stand for stromal deposits. Scale bar: $10 \mu \mathrm{m}$. 
SQSTM1/p62 immunolabeling was present in corneal epithelial cells in MCD (Fig. 4F). Fig. 4G and H represent low magnification merged microphotos for Hsp70, Ub and SQSTM1/p62 immunofluorescent reaction of corneal epithelium from healthy (Fig. 4G) and MCD corneal buttons (Fig. $4 \mathrm{H}$ ), respectively. The frames represent the areas shown in higher magnification in panels (Fig. 4G1,H1). On Fig. 4H1 around the yellow (artificial color chosen for DAPI) nucleus pink dots may indicate the colocalization of Hsp70, Ub and SQSTM1/p62.

The very low autofluorescent signal on Figure $5 \mathrm{~F}$
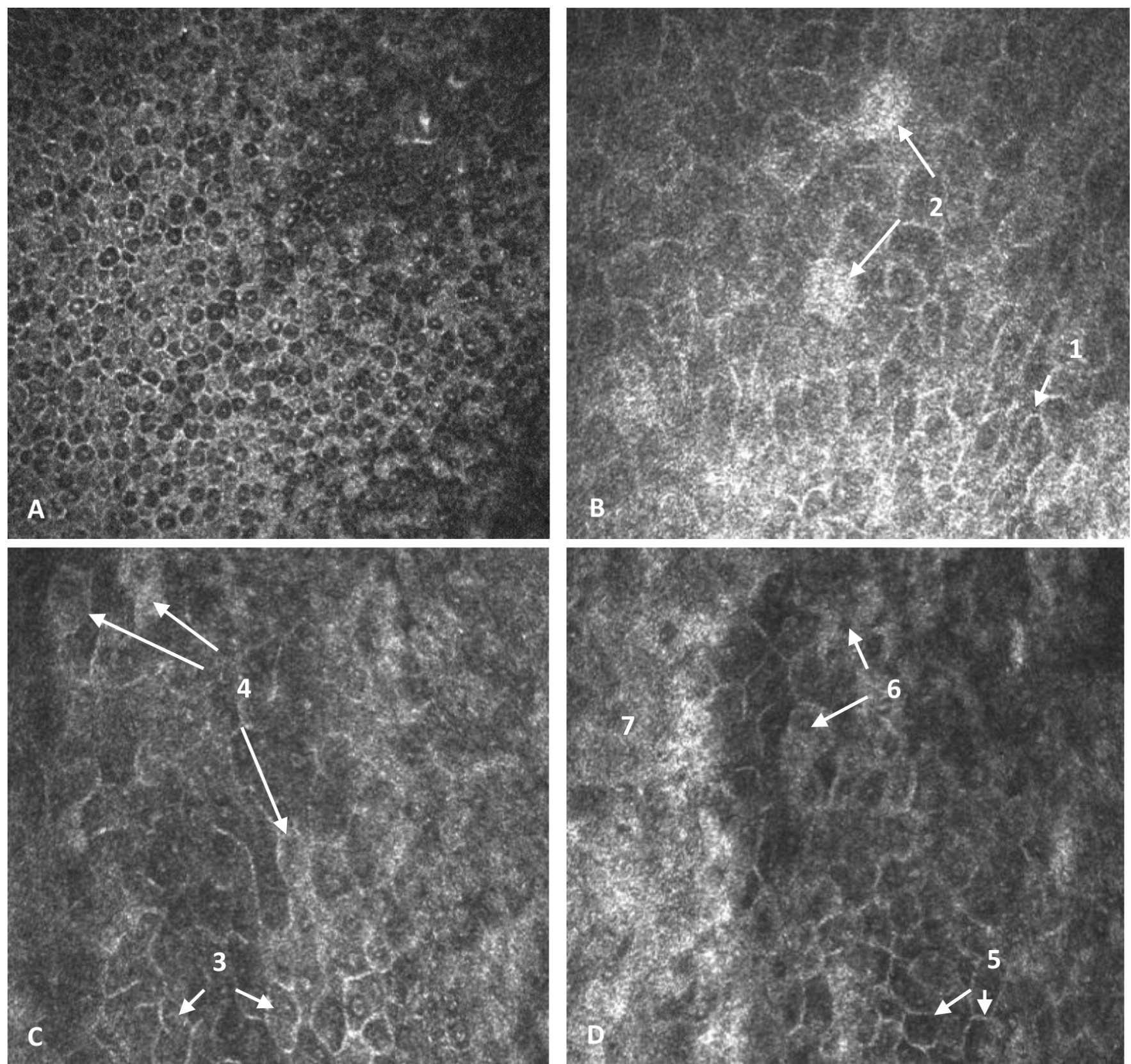

Fig. 2. Confocal microscopy analysis from epithelium $(300 \times 300 \mu \mathrm{m}$, depth $\sim 20 \mu \mathrm{m})$. A. Healthy control is shown in the left upper panel. B. In initial dystrophy state abnormality in cell size and shape can be observed. Cells become bigger and polygonal with bright contours (1). Single cells show intracellular deposits with saved cellular shape (2). C. In moderate MCD state still visible bright contoured, polygonal cells (3) with increased number of deposits accumulate cells (4), shape of cells is still saved and no leaking out is observed. D. In severe MCD state there are single bright contoured cells visible (5), as well as cells with deposits and saved shape (6) but the intensity of changes is higher. 
indicating the present of intact Bowman membrane (BM) of the healthy cornea button (dash line) compared to the MCD samples, where due to the absent of $\mathrm{BM}$ there was no signal observed (Fig. 5A). Note that the stromal cells from MCD corneal buttons showed weak Hsp70 positivity (Fig 5B, green), in association with moderate Ub (Fig. 5C, red) and SQSTM1/p62 (Fig. 5D, blue) immunolabelling. On the merged panel of Figure
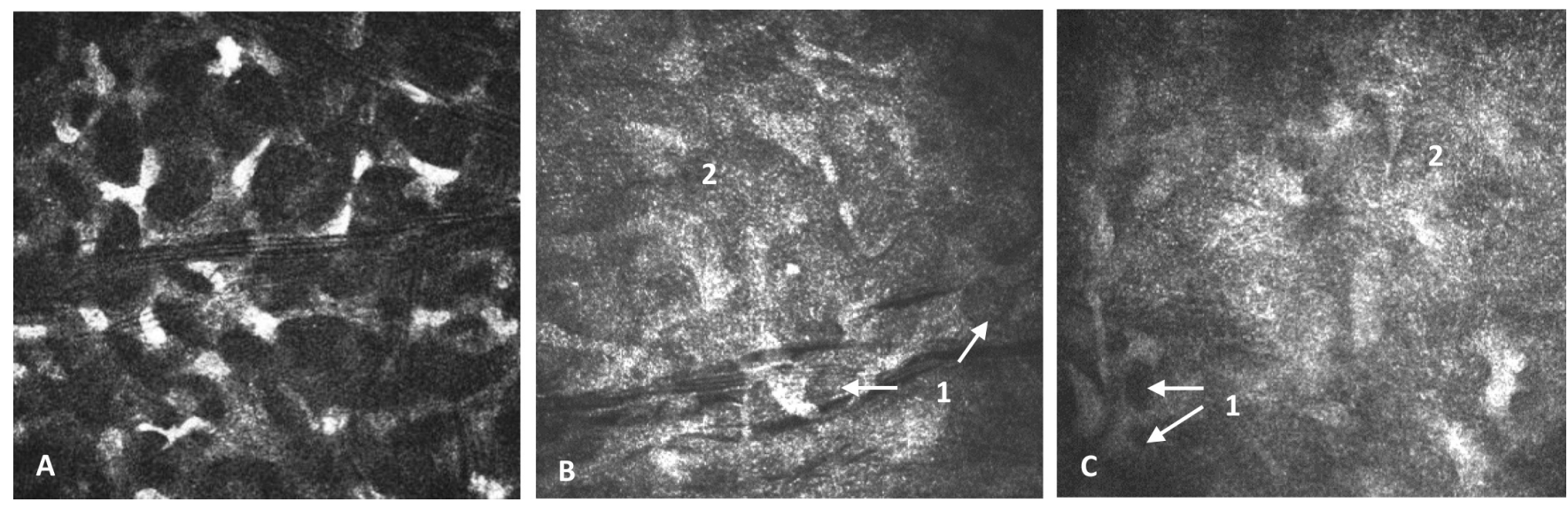

Fig. 3. Confocal microscopy analysis from anterior stroma (300x300 $\mu \mathrm{m}$, depth $\sim 150 \mu \mathrm{m})$. A. anterior stroma of healthy cornea with regular, condensed keratocytes, small fibrilles, no vacuoles, dark background from high transparency stromal matrix. $\mathbf{B}$ and $\mathbf{C}$. Hyperreflective keratocytes with presence of vacuoles (1) - proof of accumulative activity in MCD, bright, homogenous background from stromal matrix cause blurring in MCD (2).
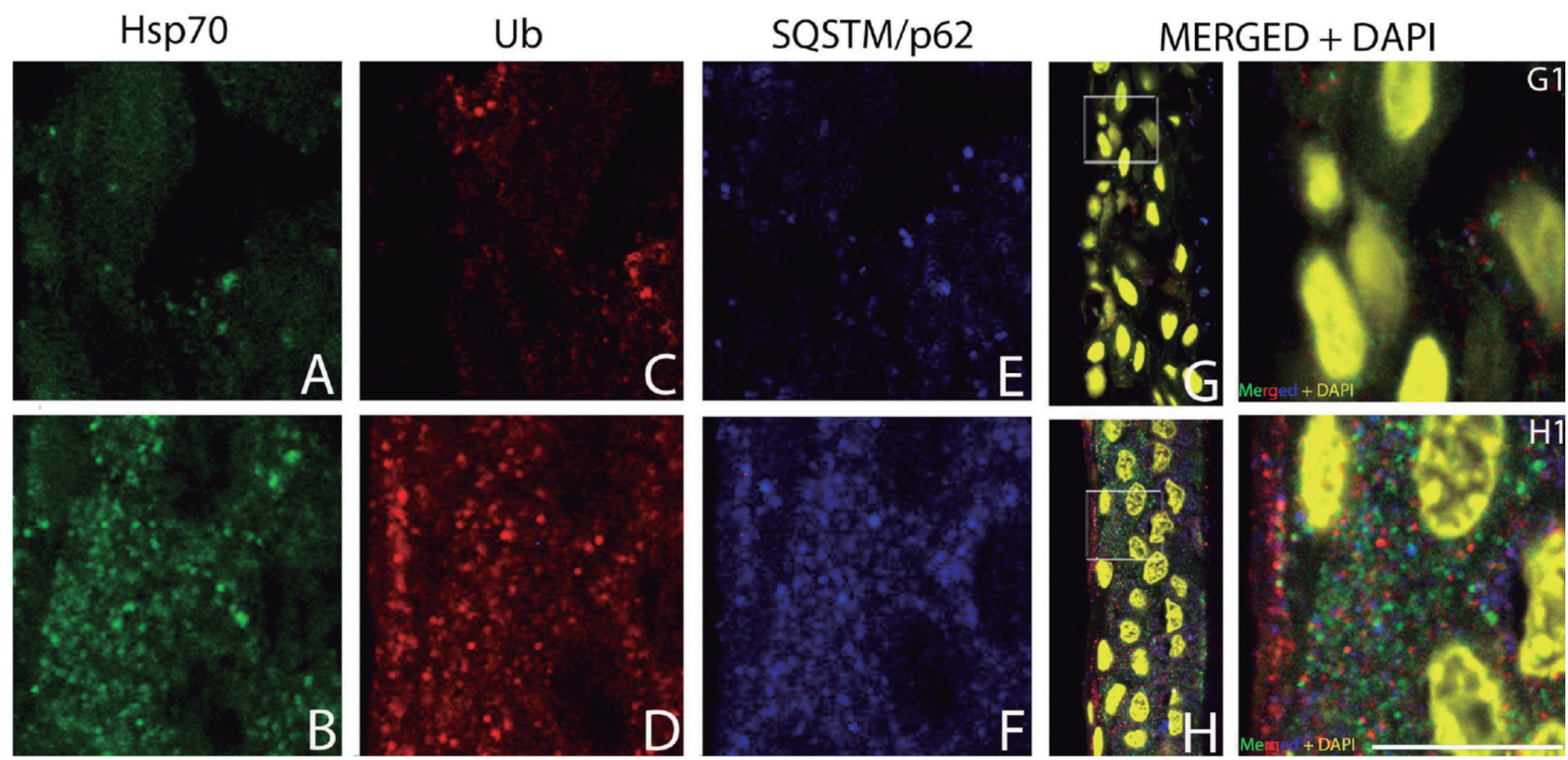

Fig. 4. High magnification microphotos of single laser scanning confocal optical sections of corneal basal epithelial cells layer ( $1 \mu \mathrm{m})$. Control samples (upper panel) and MCD samples (lower panel) demonstrate the immunostaining of the anti-Hsp70 (A, B, green) and anti-ubiquitin-2 (C, D, red) and anti-SQSTM1/p62 (E, F, blue) antibodies in corneal basal epithelial cells. $\mathbf{G}$ and $\mathbf{H}$ represent low magnification merged microphotos for Hsp70, Ub protein conjugates and SQSTM1/p62 immunofluorescent reaction of corneal epithelium from healthy $(\mathbf{G})$ and $M C D$ corneal buttons $(\mathbf{H})$. The frames represent the areas $(18 \times 18 \mu \mathrm{m})$ shown in higher magnification in panels (G1), (H1). Triple immunofluorescence labeling of Hsp70, Ub protein conjugates, and SQSTM1/p62 combined with DAPI (yellow) illustrates definitive labeling for these molecules in MCD cases. Note that no nuclear staining is present on the merged images of $\mathbf{G} \mathbf{1}$ and $\mathbf{H 1}$ preclude the possibility of nonspecific reaction (internal control). On $\mathbf{H} \mathbf{1}$ around the yellow (artificial color choosen for DAPI) nucleus pink dots may indicate the colocalization of these antibodies used. A-F, x 600; Scale bar: $10 \mu \mathrm{m}$. 

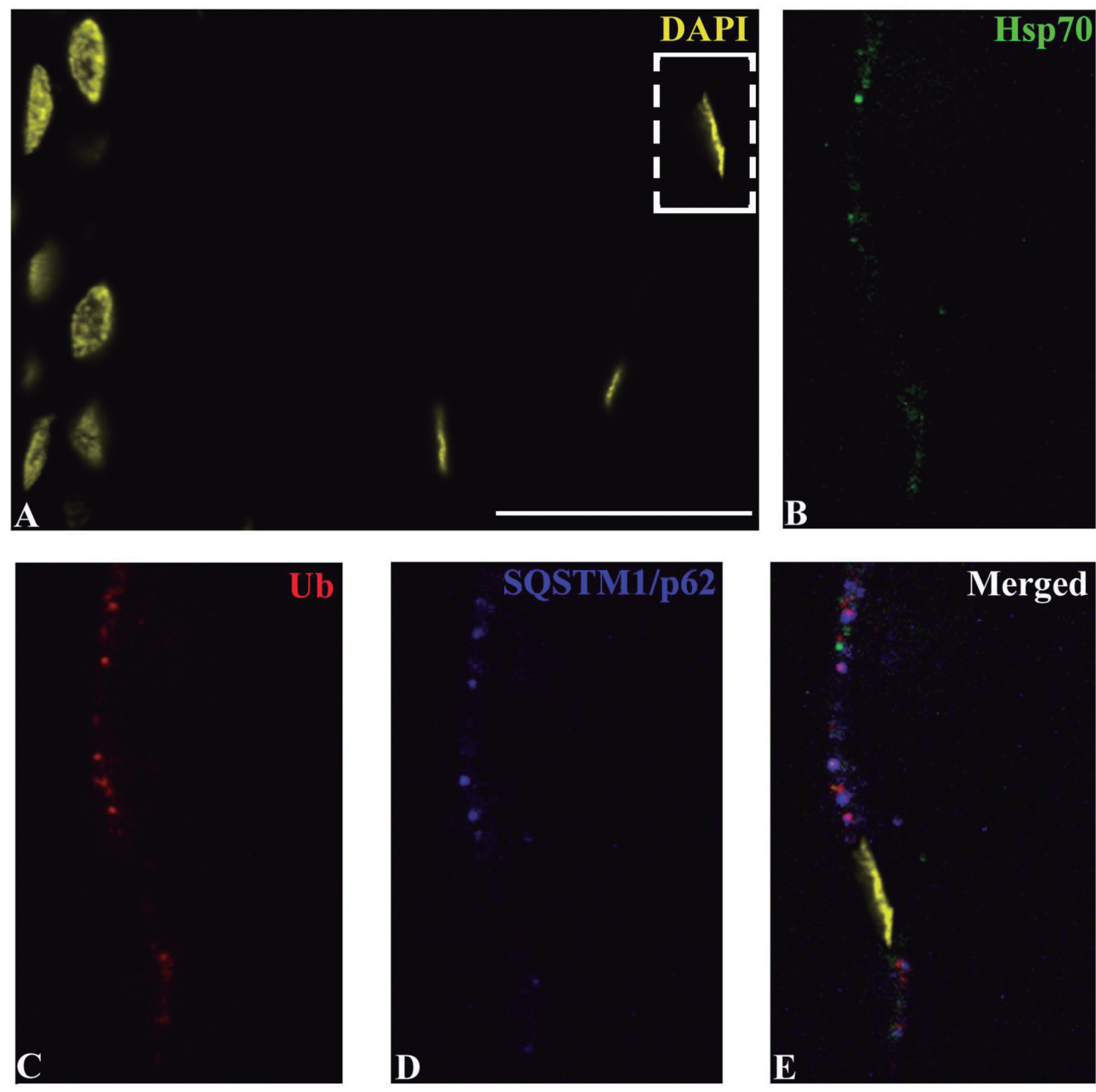

Fig. 5. High

magnification

microphotos of single

laser scanning

confocal optical

sections showing

border of corneal

basal epithelial cell

layer and anterior

corneal stroma (1

$\mu \mathrm{m})$. DAPI positive

epithelial (round) and

stromal (elongated)

cells nuclei (yellow)

are visible in low

magnification picture

from MCD (A) and

healthy $(F)$ samples

respectively. The

square in $\mathbf{A}$ indicates

high magnification

area $(4 \times 6.5 \mu \mathrm{m})$,

shown in $\mathbf{B}-\mathbf{E}$.

respectively. The

very low

autofluorescent

signal indicating the

present of intact

Bowman membrane

(BM) of the healthy

cornea button (dash

line) compared to the

MCD samples,

where due to the

absence of BM there

was no signal

observed. Note that

the stromal cells

from MCD corneal

buttons showed

weak Hsp70

positivity (B, green),

in association with

moderate $\mathrm{Ub}$ protein

conjugates (C, red)

and SQSTM/p62 (D,

blue)

immunolabelling. On

the merged panel the

colocalization of

these proteins on the

"pseudopod-like"

processes of stomal

cells extending

parallel to the basal

epithelial cells is

obvious (E). Scale 


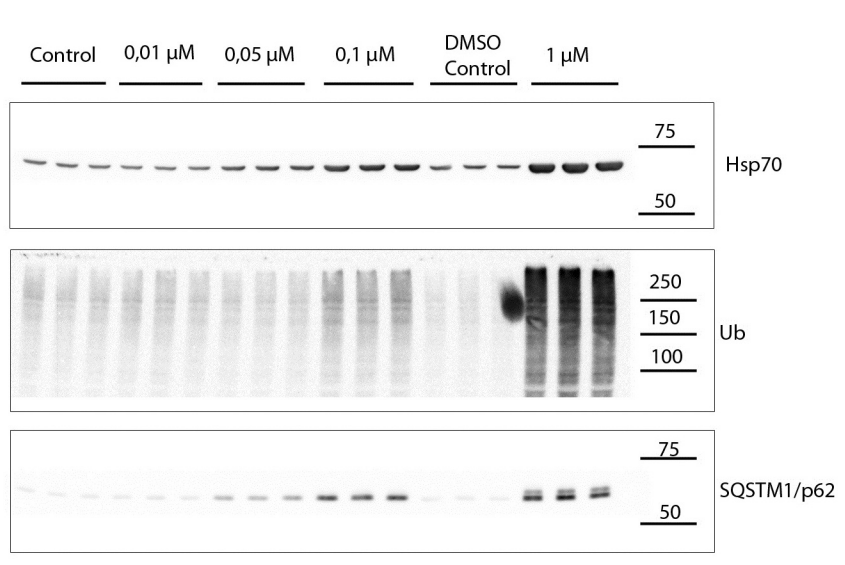

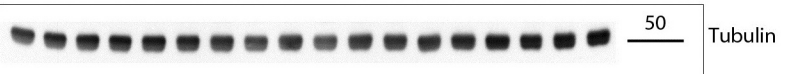

Fig. 6. Proteasome inhibition upregulates Hsp70, ubiquitin protein conjugates and SQSTM1/p62 in HCE-2 cells. Western blotting analysis for evaluating the effects of different concentrations of protesome inhibition (MG-132). Exposure for 24 hours on expression of Hsp70, Ub protein conjugates and SQSTM1/p62. a-tubulin staining was used to ensure equal loading of proteins.
$5 \mathrm{E}$ the colocalization of these proteins on the "pseudopod-like" processes of stomal cells extending parallel to the basal epithelial cells can be observed.

Since in vivo data suggested impaired proteasomal clearance at both basal corneal epithelium and stromal keratocytes, we wanted to examine the effect of a proteasome inhibitor in human HCE-2 corneal epithelial cells in vitro. As shown in Figure 6, Hsp70, Ub conjugates and SQSTM1/p62 protein levels strongly increased in a concentration gradient dependent way in response to MG-132 proteasome inhibitor in HCE-2 cells. This protein upregulation was accompanied by perinuclear protein aggregation, when cells were exposed to MG-132 proteasome inhibition and analyzed by a transmission electron microscope (Fig.7).

\section{Discussion}

Protein misfolding may promote cellular degeneration and cell death (Kopito, 2000; Estey et al., 2010; Underhaug et al., 2013, Viiri et al., 2013). All cells have special surveillance systems to control steady-state of the folding and clearance of proteins. Hsps are the
A
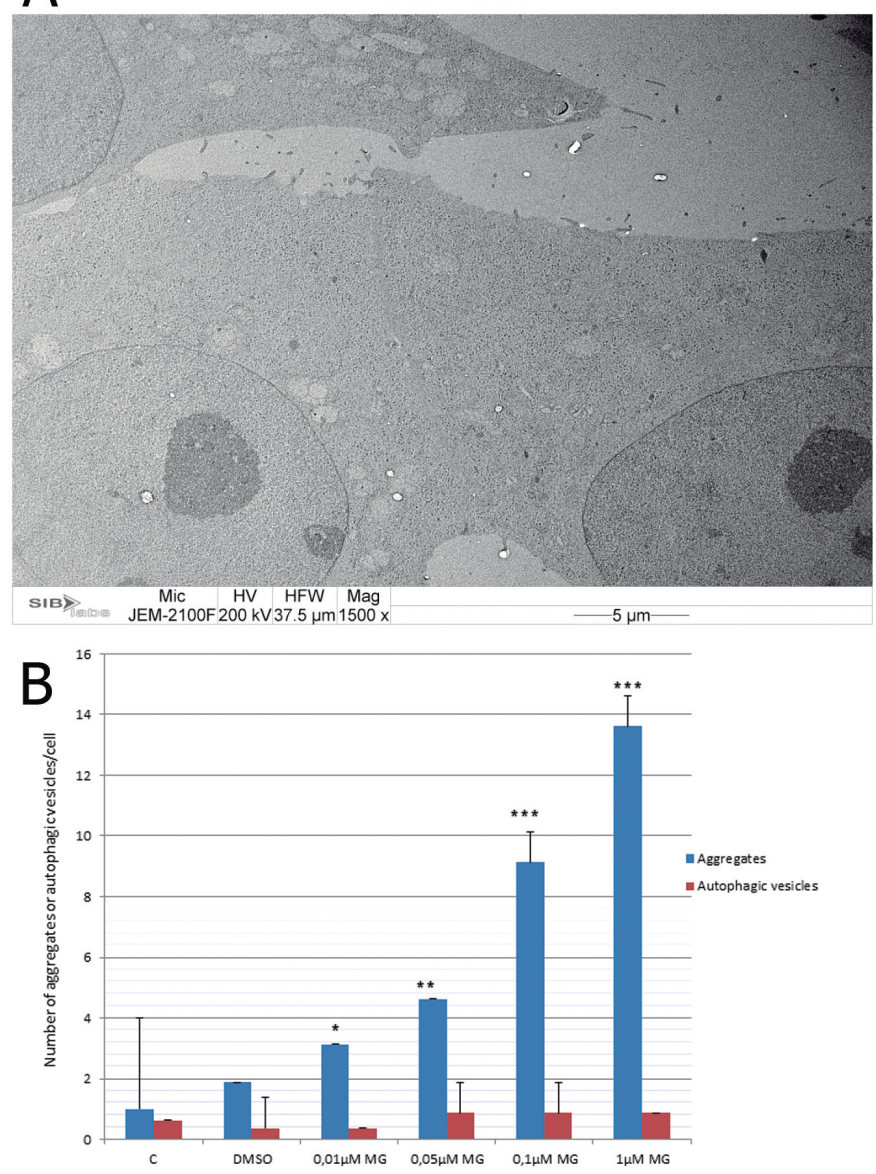

$1 \mu \mathrm{M}$ MG-132

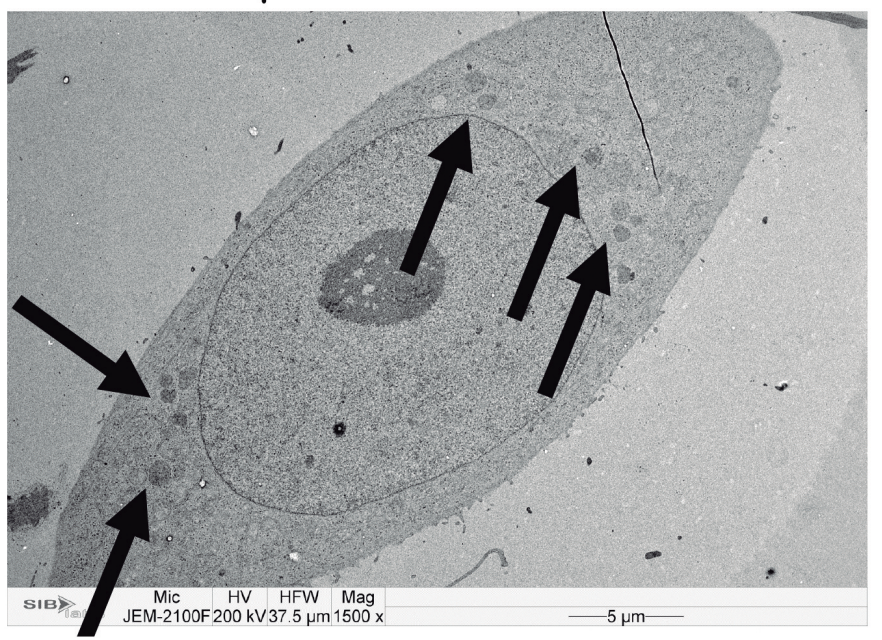

Fg. 7. Proteasome inhibition increases perinuclear aggregates. A. Transmission electron microscopy of control HCE-2 cells and from cells exposed to $1 \mu \mathrm{M} \mathrm{MG}-132$ for 24 hours. B. Quantification of aggregates per cell. Eight parallel samples were measured in all treatments. 


\section{Proteotoxic stress in macular dystrophy}

only system in cells to repair misfolding proteins and release them as a functional unit (Hartl and Hayer-Hartl, 2009). They assist in the refolding of damaged proteins, facilitate their translocation to their correct intracellular localization and attempt to prevent aggregation of proteins (Arrigo and Simon, 2010). If Hsps capacity to refold proteins is exceeded damaged proteins are targeted to UPP clearance machine (Jung et al., 2009). UPP is the master proteolytic system in the cells that clean misfolded Ub-labelled soluble proteins. Moreover, lysosomal autophagy is integrated with proteasomal degradation system (Ryhänen et al., 2009; Viiri et al., 2013). In this study, we show that Hsp70 is accumulated in both basal epithelium and stromal cells. A previous immunohistochemical study indicates increased apoptosis of epithelial cells, keratocytes and endothelial cells in MCD (Szentmáry et al., 2007). Hsp70 has a strong anti-apoptotic capacity (Beere et al., 2000; Saleh et al., 2000; Ravagnan et al., 2001). Thus, upregulation of Hsp70 reveals an attempt to repair misfolded proteins and prevent cell death in MCD.

The SQSTM1/p62 was first characterized in polyubiquitinated protein aggregates in response to proteasomal depletion (Kuusisto et al., 2001). The ubiquitin-associated domain (UBA) of SQSTM1/p62 enables a non-covalent binding to ubiquitin or ubiquitinated substrate proteins that are then targeted to proteasomal degradation, where the N-terminal PB 1 domain of p62 binds to the proteasome (Seibenhener et al., 2004; Babu et al., 2005). Alternatively, ubiquitinated complexes are shuttled to autophagocytic degradation (Ryhänen et al., 2009; Viiri et al., 2013; Komatsu et al., 2007; Pankiv et al., 2007; Kaarniranta et al., 2013). Decreased proteasomal activity evokes Hsp70, Ub protein conjugates and SQSTM1/p62 accumulation in cells (Ryhänen et al., 2009; Viiri et al., 2010). We observed a strong upregulation of Ub protein conjugates and SQSTM1/p62 in both the basal epithelial cells and the stromal keratocytes in MCD samples. This discovery was supported by the western blot and TEM analysis under proteasome inhibition in HCE-2 cells in vitro. Although Hsp70, Ub protein conjugates and SQSTM1/p62 localize to the same protein aggregation area, it seems that Hsp70 differs in its function in proteolysis. It is highly probable that the Hsp70 participates for protein folding processes, while $\mathrm{Ub}$ and SQSTM1/p62 regulate clearance systems (Viiri et al., 2010). Accumulated Ub protein conjugates and SQSTM1 may also be a reflection of impaired autophagy (Choi et al., 2012). Our recent observation reveals that Hsp70 does not undergo autophagy clearance (Kivinen et al., 2014). Since, Hsp70 is upregulated in MCD samples it reveals at least partly impaired proteasomal function in the pathology.

Understanding the intricate network of pathways that maintain proteostasis and mediate stress response or default into cell death is critical to understand MCD pathology. We suggest a novel mechanism for the pathogenesis of MCD that involves impaired proteasomal clearance in both the corneal basal epithelium and the stromal cells. Impaired cellular proteolysis may also coincide with an increased unfolding protein stress response in the endoplasmic reticulum that disturbs protein processing and leads finally to different deposit accumulations (Digaleh et al., 2013), as it occurs in MCD.

Acknowledgements. The authors would like to thank Anne Seppänen, Anne Mari Haapaniemi, Tünde Terdik and Chang Hwa Ham for excellent technical assistance. EVO grants of Kuopio University Hospital, the Finnish Cultural Foundation and its North Savo Fund, the Finnish Eye Foundation, the Finnish Funding Agency for Technology and Innovation, Health Research Council of the Academy of Finland, Päivikki and Sakari Sohlberg Foundation and a support from University of Debrecen (RH/885/2013) and from the European Union and the State of Hungary, co-financed by the European Social Fund in the framework of TÁMOP 4.2.4. A/2-11-1-2012-0001 is acknowledged. We thank for the support of Bolyai János and John von Neumann Research Scholarship and the high-quality manuscript service of American Journal Experts (AJE).

\section{References}

Akama T.O., Nishida K., Nakayama J., Watanabe H., Ozaki K., Nakamura T., Dota A., Kawasaki S., Inoue Y., Maeda N., Yamamoto S., Fujiwara T., Thonar E.J., Shimomura Y., Kinoshita S., Tanigami A. and Fukuda M.N. (2000). Macular corneal dystrophy type I and type II are caused by distinct mutations in a new sulphotransferase gene. Nat. Genet. 26, 237-241.

Arrigo A.P. and Simon S. (2010). Expression and functions of heat shock proteins in the normal and pathological mammalian eye. Curr. Mol. Med. 10, 776-793.

Babu J.R., Geetha T. and Wooten W. (2005). Sequestosome 1/p62 shuttles polyubiquitinated tau for proteasome degradation. J. Neurochem. 94, 192-203.

Beere H.M., Wolf B.B., Cain K., Mosser D.D., Mahboubi A., Kuwana T., Tailor P., Morimoto R.I., Cohen G.M. and Green D.R. (2000). Heatshock protein 70 inhibits apoptosis by preventing recruitment of procaspase-9 to the Apaf-1 apoptosome. Nat. Cell Biol. 2, 469-475.

Carrard G., Bulteau A.L., Petropoulos I. and Friguet B. (2002). Impairment of proteasome structure and function in aging. Int. J. Biochem. Cell Biol. 34, 1461-1474.

Choi S.I., Kim B.Y., Dadakhujaev S., Oh J.Y., Kim T.I., Kim J.Y. and Kim E.K. (2012). Impaired autophagy and delayed autophagic clearance of transforming growth factor $\beta$-induced protein (TGFBI) in granular corneal dystrophy type 2. Autophagy 8, 1782-1797.

Digaleh H., Kiaei M. and Khodagholi F. (2013). Nrf2 and Nrf1 signaling and ER stress crosstalk: implication for proteasomal degradation and autophagy. Cell Mol. Life Sci. 70, 4681-4694

Estey T., Chen Y., Carpenter J.F. and Vasiliou V. (2010). Structural and functional modifications of corneal crystallin ALDH3A1 by UVB light. PLoS One 5, e15218.

Felszeghy S., Módis L., Németh P., Nagy G., Zelles T., Agre P., Laurikkala J., Fejerskov O., Thesleff I. and Nielsen S. (2004). Expression of aquaporin isoforms during human and mouse tooth development. Arch. Oral. Biol. 49, 247-257.

Goldberg A.L. (2003). Protein degradation and protection against 


\section{Proteotoxic stress in macular dystrophy}

misfolded or damaged proteins. Nature 426, 895-899.

Hartl F.U. and Hayer-Hartl M. (2009). Converging concepts of protein folding in vitro and in vivo. Nat. Struct. Mol. Biol. 16, 574-581.

Janse D.M., Crosa B., Finley D. and Church G.M. (2004). Localization to the proteasome is sufficient for degradation. J. Biol. Chem. 279, 21415-21420.

Jonasson F., Johannsson J.H., Garner A. and Rice N.S. (1989). Macular corneal dystrophy in Iceland. Eye (Lond) 3, 446-454.

Jung T., Catalgol B. and Grune T. (2009). The proteasomal system. Mol. Aspects Med. 30, 191-296.

Kaarniranta K., Sinha D., Blasiak J., Kauppinen A., Veréb Z., Salminen A., Boulton M.E. and Petrovski G. (2013). Autophagy and heterophagy dysregulation leads to retinal pigment epithelium dysfunction and development of age-related macular degeneration. Autophagy 9, 973-984.

Kästle M. and Grune T. (2012). Interactions of the proteasomal system with chaperones: protein triage and protein quality control. Prog. Mol. Biol. Transl. Sci. 109, 113-160.

Kinnunen K., Kauppinen A., Piippo N., Koistinen A., Toropainen E. and Kaarniranta K. (2014) Cationorm shows good tolerability on human HCE-2 corneal epithelial cell cultures. Exp. Eye Res. 120, 82-89.

Kivinen N., Hyttinen J.M.T., Viiri J., Paterno J.J. Felszheghy S., Kauppinen A., Salminen A. and Kaarniranta K. (2014). Hsp70 binds reversibly to proteasome inhibitor-induced protein aggregates and evades autophagic clearance in ARPE-19 cells. J. Biochem. Pharmacol. Res. 2, 1-7

Klintworth G.K. (2009). Corneal dystrophies. Orphanet J. Rare Dis. 4, 7.

Klintworth G.K. and Smith C.F. (1977). Macular corneal dystrophy: Studies of sulfated glycosaminoglycans in corneal explant and confluent stromal cell cultures. Am. J. Pathol. 89, 167-182.

Klintworth G.K., Meyer R., Dennis R., Hewitt A.T., Stock E.L., Lenz M.E., Hassell J.R., Stark W.J. Jr, Kuettner K.E. and Thonar E.J. (1986). Macular corneal dystrophy. Lack of keratan sulfate in serum and cornea. Ophthal. Paediatr. Genet. 7, 139-143.

Klintworth G.K., Oshima E., al-Rajhi A., al-Saif A., Thonar E.J. and Karcioglu Z.A. (1997). Macular corneal dystrophy in Saudi Arabia: a study of 56 cases and recognition of a new immunophenotype. Am. J. Ophthalmol. 124, 9-18.

Komatsu M., Waguri S., Koike M., Sou Y.S., Ueno T., Hara T., Mizushima N., Iwata J., Ezaki J., Murata S., Hamazaki J., Nishito Y., lemura S., Natsume T., Yanagawa T., Uwayama J., Warabi E., Yoshida H., Ishii T., Kobayashi A., Yamamoto M., Yue Z., Uchiyama Y., Kominami E. and Tanaka K. (2007). Homeostatic levels of p62 control cytoplasmic inclusion body formation in autophagy-deficient mice. Cell 131, 1149-1163.

Kopito R.R. (2000). Aggresomes, inclusion bodies and protein aggregation. Trends Cell. Biol. 10,524-530.

Kuusisto E., Suuronen T. and Salminen A. (2001). Ubiquitin-binding protein p62 expression is induced during apoptosis and proteasomal inhibition in neuronal cells. Biochem. Biophys. Res. Commun. 280, 2 23-228.

Pankiv S., Clausen T.H., Lamark T., Brech A., Bruun J.A., Outzen H., Øvervatn A., Bjørkøy G. and Johansen T. (2007). p62/SQSTM1 binds directly to Atg8/LC3 to facilitate degradation of ubiquitinated protein aggregates by autophagy. J. Biol. Chem. 282, 24131-24145.

Ravagnan L., Gurbuxani S., Susin S.A., Maisse C., Daugas E.,
Zamzami N., Mak T., Jäättelä M., Penninger J.M., Garrido C. and Kroemer G. (2001). Heat-shock protein 70 antagonizes apoptosisinducing factor. Nat. Cell Biol. 3, 839-843.

Ryhänen T., Hyttinen J.M., Kopitz J., Rilla K., Kuusisto E., Mannermaa E., Viiri J., Holmberg C.I., Immonen I., Meri S., Parkkinen J., Eskelinen E.L., Uusitalo H., Salminen A. and Kaarniranta K. (2009). Crosstalk between Hsp70 molecular chaperone, lysosomes and proteasomes in autophagy-mediated proteolysis in human retinal pigment epithelial cells. J. Cell. Mol. Med. 13, 3616-3631.

Saint-Marie G. (1962). A paraffin embedding technique for studies employing immunofluorescence. J. Histochem. 10, 250-256.

Saleh A., Srinivasula S.M., Balkir L., Robbins P.D. and Alnemri E.S. (2000). Negative regulation of the Apaf-1 apoptosome by Hsp70. Nat. Cell Biol. 2, 476-483.

Seibenhener M.L., Babu J.R., Geetha T., Wong H.C., Krishna N.R. and Wooten M.W. (2004). Sequestosome 1/p62 is a polyubiquitin chain binding protein involved in ubiquitin proteasome degradation. Mol. Cell. Biol. 24, 8055-8068.

Szentmáry N., Takács L., Berta A., Szende B., Süveges I. and Módis L. (2007). Cell proliferation and apoptosis in stromal corneal dystrophies. Histol. Histopathol. 22, 837-845.

Szentmáry N., Stündl A., Szende B. and Süveges I. (2010). p21, p27, bax, cathepsin and survivin pathways in macular dystrophy corneas. Histol. Histopathol. 25, 287-290.

Tyedmers J., Mogk A. and Bukau B. (2010). Cellular strategies for controlling protein aggregation. Nat. Rev. Mol. Cell Biol. 11, 777788.

Underhaug J., Koldsø H., Runager K., Nielsen J.T., Sørensen C.S., Kristensen T., Otzen D.E., Karring H., Malmendal A., Schiøtt B., Enghild J.J. and Nielsen N.C. (2013). Mutation in transforming growth factor beta induced protein associated with granular corneal dystrophy type 1 reduces the proteolytic susceptibility through local structural stabilization. Biochim. Biophys. Acta 1834, 2812-2822

Vadlamudi R.K., Joung I., Strominger J.L. and Shin J. (1996). p62, a phosphotyrosine-independent ligand of the $\mathrm{SH} 2$ domain of p56lck, belongs to a new class of ubiquitin-binding proteins. J. Biol. Chem. 271, 20235-20237.

Viiri J., Hyttinen J.M., Ryhänen T., Rilla K., Paimela T., Kuusisto E., Siitonen A., Urtti A., Salminen A. and Kaarniranta K. (2010). p62/sequestosome 1 as a regulator of proteasome inhibitor-induced autophagy in human retinal pigment epithelial cells. Mol. Vis. 16, 1399-1414.

Viiri J., Amadio M., Marchesi N., Hyttinen J.M., Kivinen N., Sironen R., Rilla K., Akhtar S., Provenzani A., D'Agostino V.G., Govoni S., Pascale A., Agostini H., Petrovski G., Salminen A. and Kaarniranta K. (2013). Autophagy activation clears ELAVL1/HuR -mediated accumulation of SQSTM1/p62 during proteasomal inhibition in human ARPE-19 retinal pigment epithelial cells. PLoS One 8, e69563.

Weiss J.S., Møller H.U., Lisch W., Kinoshita S., Aldave A.J., Belin M.W., Kivelä T., Busin M., Munier F.L., Seitz B., Sutphin J., Bredrup C., Mannis M.J., Rapuano C.J., Van Rij G., Kim E.K. and Klintworth G.K. (2008). The IC3D classification of the corneal dystrophies. Cornea 27 (Suppl 2), S1-83.

Accepted January 19, 2015 